\title{
ON 2-LOCAL DIAMETER-PRESERVING MAPS BETWEEN $C(X)$-SPACES
}

\author{
A. JIMÉNEZ-VARGAS AND FERESHTEH SADY
}

\begin{abstract}
The 2-locality problem of diameter-preserving maps between $C(X)$-spaces is addressed in this paper. For any compact Hausdorff space $X$ with at least three points, we give an example of a 2-local diameter-preserving map on $C(X)$ which is not linear. However, we show that for first countable compact Hausdorff spaces $X$ and $Y$, every 2-local diameter-preserving map from $C(X)$ to $C(Y)$ is linear and surjective up to constants in some sense. This yields the 2-algebraic reflexivity of isometries with respect to the diameter norms on the quotient spaces.
\end{abstract}

\section{INTRODUCTION AND RESULTS}

Let $E$ and $F$ be Banach spaces and let $\mathcal{S}$ be a subset of $\mathcal{L}(E, F)$, the space of linear operators from $E$ to $F$. Let us recall that a linear map $T: E \rightarrow F$ is a local $\mathcal{S}$-map if for every $e \in E$, there exists a $T_{e} \in \mathcal{S}$, depending possibly on $e$, such that $T_{e}(e)=T(e)$. On the other hand, a map $\Delta: E \rightarrow F$ (which is not assumed to be linear) is called a 2-local $\mathcal{S}$-map if for any $e, u \in E$, there exists a $T_{e, u} \in \mathcal{S}$, depending in general on $e$ and $u$, such that $T_{e, u}(e)=\Delta(e)$ and $T_{e, u}(u)=\Delta(u)$.

Most of the published works on local and 2-local $\mathcal{S}$-maps concern the set $\mathcal{S}=\mathcal{G}(E)$, the group of surjective linear isometries of $E$. In this case, the local and 2-local $\mathcal{G}(E)$-maps are known as local and 2-local isometries of $E$, respectively. The main question which one raises is for which Banach spaces, every local isometry is a surjective isometry or, equivalently, which Banach spaces have an algebraically reflexive isometry group. In the 2-local setting, the basic problem is to show that every 2-local isometry is a surjective linear isometry.

In [21, Molnár initiated the study of 2-local isometries on operator algebras and proposed to research the 2-locality of isometries on function algebras. In this line, Győry [11] dealed with 2local isometries on spaces of continuous functions. In [18], Villegas and the first author adapted the Györy's technique to analyze the 2-local isometries on Lipschitz algebras. Hatori, Miura, Oka and Takagi [13] considered 2-local isometries on uniform algebras including certain algebras of holomorphic functions. More recently, Hosseini [15], Hatori and Oi [14] and Li, Peralta, L. Wang and Y.-S. Wang [20, have investigated 2-local isometries of different function algebras such as uniform algebras, Lipschitz algebras and algebras of continuously differentiable functions.

Our aim in this paper is to study the 2-locality problem for isometries between certain quotient Banach spaces which appear in a natural form when one treats with maps between $C(X)$-spaces which preserve the diameter of the range.

Let $C(X)$ be the Banach space of all continuous complex-valued functions on a compact Hausdorff space $X$, with the usual supremum norm. A map $\Delta: C(X) \rightarrow C(Y)$ (not necessarily linear) is diameter-preserving if

$$
\rho(\Delta(f)-\Delta(g))=\rho(f-g) \quad(f, g \in C(X)),
$$

where for each $f \in C(X)$,

$$
\rho(f)=\sup \{|f(x)-f(z)|: x, z \in X\} .
$$

Győry and Molnár [12] introduced this kind of maps and gave a complete description of diameterpreserving linear bijections of $C(X)$, when $X$ is a first countable compact Hausdorff space. Cabello

Date: April 15, 2020.

2010 Mathematics Subject Classification. 46B04, 47B38.

Key words and phrases. 2-local map; diameter-preserving map; function space; weighted composition operator. 
Sánchez [5] and González and Uspenskij [10] established the same characterization without the first countability assumption. As usual, $\mathbb{T}$ denotes the unit circle of $\mathbb{C}$. We also put

$$
\mathbb{T}^{+}=\left\{e^{i t}: t \in[0, \pi)\right\} .
$$

Moreover, $1_{X}$ and $0_{X}$ stand for the constant functions 1 and 0 on $X$, respectively.

Theorem 1. 5, 10, 12. Let $X$ and $Y$ be compact Hausdorff spaces. A linear bijection $T: C(X) \rightarrow$ $C(Y)$ is diameter-preserving if and only if there exist a homeomorphism $\phi: Y \rightarrow X$, a linear functional $\mu: C(X) \rightarrow \mathbb{C}$ and a number $\lambda \in \mathbb{T}$ with $\lambda \neq-\mu\left(1_{X}\right)$ such that

$$
T(f)=\lambda f \circ \phi+\mu(f) 1_{Y} \quad(f \in C(X)) .
$$

The main problem addressed in the study of diameter-preserving maps between function algebras is establishing a representation of such maps as the sum of a weighted composition operator and a functional as in Theorem 1. We have a precise description of diameter-preserving maps for most of the classical function spaces (see for example [1, 2, 3, 5, 17, 9, 23] for diameter-preserving linear maps and [4, 8, 16] for the non-linear case).

In the case in which $\mathcal{S}$ is the set of all diameter-preserving linear bijections from $C(X)$ to $C(Y)$, we studied in a recent paper [17] the local $\mathcal{S}$-maps, referred there to as local diameter-preserving maps. Namely, we proved that in the case where $X$ and $Y$ are first countable, every local diameterpreserving map from $C(X)$ to $C(Y)$ is a diameter-preserving bijection. The first countability on the topological spaces is a mild and appropriate condition when one addresses these problems. For example, the isometry group and the automorphism group of $C(X)$ are algebraically reflexive in case $X$ is first countable [22], but that reflexivity fails if $X$ is not (see section 7 in [6]).

It is natural to arise the corresponding question in the 2-local context, that is, is every 2-local diameter-preserving map a diameter-preserving linear bijection? Unfortunately or not, the answer is negative as we see next with a counterexample.

Let us recall that a map $\Delta: C(X) \rightarrow C(Y)$ (not assumed to be linear) is a 2-local diameterpreserving map if for any $f, g \in C(X)$, there exists a diameter-preserving linear bijection $T_{f, g}$ from $C(X)$ to $C(Y)$ such that $T_{f, g}(f)=\Delta(f)$ and $T_{f, g}(g)=\Delta(g)$.

Example. (A 2-local diameter-preserving non-linear map between $C(X)$-spaces). Let $X$ and $Y$ be homeomorphic compact Hausdorff spaces with at least three points. Let $\phi: Y \rightarrow X$ be a homeomorphism and let $\mu: C(X) \rightarrow \mathbb{C}$ be a homogeneous non-additive functional such that $\mu\left(1_{X}\right) \neq-1$ and $\mu\left(1_{X}-f\right)=\mu\left(1_{X}\right)-\mu(f)$ for all $f \in C(X)$. To give an example of such a functional $\mu$, fix three distinct points $x_{1}, x_{2}, x_{3} \in X$ and define $\mu: C(X) \rightarrow \mathbb{C}$ by

$$
\mu(f)= \begin{cases}f\left(x_{1}\right) & \text { if } f\left(x_{1}\right)=f\left(x_{2}\right) \text { and } f\left(x_{1}\right) \neq f\left(x_{3}\right), \\ f\left(x_{3}\right) & \text { otherwise. }\end{cases}
$$

It is easy to see that $\mu$ is homogeneous and $\mu\left(1_{X}-f\right)=\mu\left(1_{X}\right)-\mu(f)$ for all $f \in C(X)$. Meanwhile, $\mu$ is not additive, since we can take $f, g \in C(X)$ such that $f\left(x_{1}\right)=f\left(x_{2}\right)=1$ and $f\left(x_{3}\right)=0$ and also $g\left(x_{1}\right)=g\left(x_{3}\right)=1$ and $g\left(x_{2}\right)=0$, and then $\mu(f+g)=1 \neq 2=\mu(f)+\mu(g)$.

Define now the map $\Delta: C(X) \rightarrow C(Y)$ by

$$
\Delta(f)=f \circ \phi+\mu(f) 1_{Y} \quad(f \in C(X)) .
$$

For each pair $f, g \in C(X)$, consider a linear functional $\mu_{f, g}: C(X) \rightarrow \mathbb{C}$ satisfying

$$
\mu_{f, g}(f)=\mu(f), \quad \mu_{f, g}(g)=\mu(g), \quad \mu_{f, g}\left(1_{X}\right)=\mu\left(1_{X}\right) .
$$

Notice that such a functional $\mu_{f, g}$ exists. Indeed, if $\left\{f, g, 1_{X}\right\}$ is linearly independent, the existence of $\mu_{f, g}$ can be established by extending linearly to $C(X)$ a convenient linear functional defined on $\operatorname{span}\left\{f, g, 1_{X}\right\}$. If $\left\{f, g, 1_{X}\right\}$ is linearly dependent and $1_{X} \in \operatorname{span}\{f, g\}$, then we can find a linear functional $\mu_{f, g}$ on $C(X)$ such that $\mu_{f, g}(f)=\mu(f)$ and $\mu_{f, g}(g)=\mu(g)$ (note that $\mu$ is homogeneous). Since $1_{X}=a f+b g$ for some $a, b \in \mathbb{C}$, the hypotheses on $\mu$ easily imply that $\mu_{f, g}\left(1_{X}\right)=\mu\left(1_{X}\right)$, 
as desired. In the case where $\left\{f, g, 1_{X}\right\}$ is linearly dependent and $1_{X} \notin \operatorname{span}\{f, g\}$ we conclude that $f$ and $g$ are linearly dependent and we may assume that $f=c g$ for some scalar $c$. In this case, there exists a linear functional $\mu_{f, g}$ on $C(X)$ such that $\mu_{f, g}\left(1_{X}\right)=\mu\left(1_{X}\right)$ and $\mu_{f, g}(g)=\mu(g)$. Hence $\mu_{f, g}(f)=\mu(f)$ since $\mu$ is homogeneous. Thus in each case we can find a linear functional $\mu_{f, g}: C(X) \rightarrow \mathbb{C}$ with the desired properties.

Finally, for any $f, g \in C(X)$, define $T_{f, g}: C(X) \rightarrow C(Y)$ by

$$
T_{f, g}(h)=h \circ \phi+\mu_{f, g}(h) 1_{Y} \quad(h \in C(X)) .
$$

Then $T_{f, g}$ is a diameter-preserving linear bijection by Theorem 1, Clearly, for any $f, g \in C(X)$, we have $T_{f, g}(f)=\Delta(f)$ and $T_{f, g}(g)=\Delta(g)$. Hence $\Delta$ is a 2-local diameter-preserving map which is homogeneous but not additive.

However, we shall show here that, in the case where $X$ and $Y$ are first countable, every 2-local diameter-preserving map (which is immediately diameter-preserving) is linear and surjective up to constants in some sense. Our approach consists in analysing the 2-local isometries of the following quotient Banach spaces which appear closely related to diameter-preserving maps.

Given a compact Hausdorff space $X$, let $C_{\rho}(X)$ denote the quotient space $C(X) / \operatorname{ker}(\rho)$. Clearly, $C_{\rho}(X)$ is a Banach space with the norm

$$
\left\|\pi_{X}(f)\right\|_{\rho}=\rho(f) \quad(f \in C(X)),
$$

where $\pi_{X}: C(X) \rightarrow C_{\rho}(X)$ is the canonical quotient surjection. Let us recall that a mapping $T: C_{\rho}(X) \rightarrow C_{\rho}(Y)$ (not taken linear nor surjective) is an isometry whenever

$$
\left\|T\left(\pi_{X}(f)\right)-T\left(\pi_{X}(g)\right)\right\|_{\rho}=\left\|\pi_{X}(f)-\pi_{X}(g)\right\|_{\rho} \quad(f, g \in C(X)) .
$$

Our main result is the following theorem on 2-local isometries between $C_{\rho}(X)$-spaces.

Theorem 2. Let $X$ and $Y$ be first countable compact Hausdorff spaces and let $T: C_{\rho}(X) \rightarrow C_{\rho}(Y)$ be a 2-local isometry. Then $T$ is a surjective linear isometry.

\section{Proofs}

The first key tool to prove Theorem 2 is the fact that every isometry $T$ between $C_{\rho}(X)$-spaces induces a convenient (injective) diameter-preserving map $\Delta$ between the corresponding $C(X)$ spaces which is linear or surjective if so is $T$. Towards this end, fix two points $u_{0} \in X$ and $w_{0} \in Y$ and consider the linear bijections

$$
\Psi_{X}: C(X) \rightarrow C_{\rho}(X) \oplus \mathbb{C}, \quad \Psi_{X}(f)=\left(\pi_{X}(f), f\left(u_{0}\right)\right) \quad(f \in C(X))
$$

and

$$
\Psi_{Y}: C(Y) \rightarrow C_{\rho}(Y) \oplus \mathbb{C}, \quad \Psi_{Y}(g)=\left(\pi_{Y}(g), g\left(w_{0}\right)\right) \quad(g \in C(Y)) .
$$

Lemma 1. Let $X$ and $Y$ be compact Hausdorff spaces and let $T: C_{\rho}(X) \rightarrow C_{\rho}(Y)$ be an isometry. Then $\Delta: C(X) \rightarrow C(Y)$ defined by

$$
\Delta(f)=\Psi_{Y}^{-1}\left(T\left(\pi_{X}(f)\right), f\left(u_{0}\right)\right) \quad(f \in C(X))
$$

is an injective diameter-preserving map. Moreover, $T$ is linear (respectively, surjective) if and only if so is $\Delta$.

Proof. Given $f, g \in C(X)$, we put $h=\Delta(f)-\Delta(g)$. Then

$$
\begin{aligned}
h & =\Psi_{Y}^{-1}\left(T\left(\pi_{X}(f)\right), f\left(u_{0}\right)\right)-\Psi_{Y}^{-1}\left(T\left(\pi_{X}(g)\right), g\left(u_{0}\right)\right) \\
& =\Psi_{Y}^{-1}\left(T\left(\pi_{X}(f)\right)-T\left(\pi_{X}(g)\right), f\left(u_{0}\right)-g\left(u_{0}\right)\right) .
\end{aligned}
$$

Hence

$$
\left(\pi_{Y}(h), h\left(w_{0}\right)\right)=\Psi_{Y}(h)=\left(T\left(\pi_{X}(f)\right)-T\left(\pi_{X}(g)\right), f\left(u_{0}\right)-g\left(u_{0}\right)\right),
$$


and consequently $\pi_{Y}(h)=T\left(\pi_{X}(f)\right)-T\left(\pi_{X}(g)\right)$. This implies that

$$
\begin{aligned}
\rho(\Delta(f)-\Delta(g)) & =\left\|\pi_{Y}(h)\right\|_{\rho}=\left\|T\left(\pi_{X}(f)\right)-T\left(\pi_{X}(g)\right)\right\|_{\rho} \\
& =\left\|\pi_{X}(f)-\pi_{X}(g)\right\|_{\rho} \\
& =\rho(f-g),
\end{aligned}
$$

that is, $\Delta$ is diameter-preserving. Clearly, $\Delta$ is injective. It is also easy to see that $\Delta$ is linear if so is $T$. Assume now that $T$ is surjective. Then, given $g \in C(Y)$ there exists $f \in C(X)$ such that $T\left(\pi_{X}(f)\right)=\pi_{Y}(g)$. Replacing $g$ by $g+\lambda$ for some $\lambda \in \mathbb{C}$, we can assume that $g\left(w_{0}\right)=f\left(u_{0}\right)$. Hence

$$
\Delta(f)=\Psi_{Y}^{-1}\left(T\left(\pi_{X}(f)\right), f\left(u_{0}\right)\right)=\Psi_{Y}^{-1}\left(\pi_{Y}(g), g\left(w_{0}\right)\right)=g,
$$

which shows that $\Delta$ is surjective, as well. A similar reasoning justifies that if $\Delta$ is linear (respectively, surjective), then so is $T$.

Now, we prove our main theorem.

Proof. (Theorem 2). Let $T: C_{\rho}(X) \rightarrow C_{\rho}(Y)$ be a 2-local isometry. The proof will be carried out through a series of claims. The proofs of some of them are similar to those of the corresponding steps in the proof of the main theorem (Theorem 2) of [17]. For this reason we shall only include here the proof of those claims whose arguments differ essentially from similar steps in [17].

Claim 1. The map $\Delta: C(X) \rightarrow C(Y)$ defined by

$$
\Delta(f)=\Psi_{Y}^{-1}\left(T\left(\pi_{X}(f)\right), f\left(u_{0}\right)\right) \quad(f \in C(X))
$$

is a 2-local diameter-preserving map.

Let $f, g \in C(X)$. By hypotheses, there exists a surjective linear isometry $T_{f, g}: C_{\rho}(X) \rightarrow C_{\rho}(Y)$ such that $T_{f, g}\left(\pi_{X}(f)\right)=T\left(\pi_{X}(f)\right)$ and $T_{f, g}\left(\pi_{X}(g)\right)=T\left(\pi_{X}(g)\right)$. Define $\Delta_{f, g}: C(X) \rightarrow C(Y)$ by

$$
\Delta_{f, g}(h)=\Psi_{Y}^{-1}\left(T_{f, g}\left(\pi_{X}(h)\right), h\left(u_{0}\right)\right) \quad(h \in C(X)) .
$$

By Lemma 1, $\Delta_{f, g}$ is a diameter-preserving linear bijection from $C(X)$ to $C(Y)$ satisfying $\Delta_{f, g}(f)=$ $\Delta(f)$ and $\Delta_{f, g}(g)=\Delta(g)$.

The following fact will be used repeatedly without any explicit mention in our proof.

Claim 2. For any $f, g \in C(X)$, there exists a diameter-preserving linear bijection $\Delta_{f, g}$ of $C(X)$ to $C(Y)$ such that $\Delta_{f, g}(f)=\Delta(f)$ and $\Delta_{f, g}(g)=\Delta(g)$. Moreover, there exist a homeomorphism $\phi_{f, g}: Y \rightarrow X$, a linear functional $\mu_{f, g}$ on $C(X)$ and a number $\lambda_{f, g} \in \mathbb{T}$ with $\lambda_{f, g} \neq-\mu_{f, g}\left(1_{X}\right)$ such that

and

$$
\Delta(f)(y)=\lambda_{f, g} f\left(\phi_{f, g}(y)\right)+\mu_{f, g}(f) \quad(y \in Y)
$$

$$
\Delta(g)(y)=\lambda_{f, g} g\left(\phi_{f, g}(y)\right)+\mu_{f, g}(g) \quad(y \in Y) .
$$

It follows from Claim 1 and Theorem 1 .

Claim 3. $\Delta$ is injective, diameter-preserving and homogeneous.

Let $f, g \in C(X)$. If $\Delta(f)=\Delta(g)$, then $f=g$ by the injectivity of $\Delta_{f, g}$ and therefore $\Delta$ is injective. Clearly, $\Delta$ is diameter-preserving because

$$
\rho(\Delta(f)-\Delta(g))=\rho\left(\Delta_{f, g}(f)-\Delta_{f, g}(g)\right)=\rho(f-g) .
$$

Finally, given $\lambda \in \mathbb{C}$, we have

$$
\Delta(\lambda f)=\Delta_{f, \lambda f}(\lambda f)=\lambda \Delta_{f, \lambda f}(f)=\lambda \Delta(f),
$$

and thus $\Delta$ is homogeneous. 
By Claim 2, there exists a homeomorphism from $Y$ onto $X$. Hence $Y$ and $X$ have the same cardinality. Since Theorem 2 is quite easy to verify when $Y$ is a singleton, we suppose from now on that $X$ and $Y$ have at least two points.

Given a set $X$ with cardinal number $|X| \geq 2$, we set

$$
\begin{aligned}
\widetilde{X} & =\left\{\left(x_{1}, x_{2}\right) \in X \times X: x_{1} \neq x_{2}\right\}, \\
X_{2} & =\left\{\left\{x_{1}, x_{2}\right\}:\left(x_{1}, x_{2}\right) \in \widetilde{X}\right\},
\end{aligned}
$$

and we define the natural correspondence $\Lambda_{X}: \widetilde{X} \rightarrow X_{2}$ by

$$
\Lambda_{X}\left(\left(x_{1}, x_{2}\right)\right)=\left\{x_{1}, x_{2}\right\} \quad\left(\left(x_{1}, x_{2}\right) \in \tilde{X}\right) .
$$

Given a compact Hausdorff space $X$ and a point $\left(x_{1}, x_{2}\right) \in \tilde{X}$, the Urysohn's lemma guarantees the existence of a continuous function $h_{\left(x_{1}, x_{2}\right)}: X \rightarrow[0,1]$ such that

$$
h_{\left(x_{1}, x_{2}\right)}\left(x_{1}\right)-h_{\left(x_{1}, x_{2}\right)}\left(x_{2}\right)=\rho\left(h_{\left(x_{1}, x_{2}\right)}\right) .
$$

In fact, $h_{\left(x_{1}, x_{2}\right)}\left(x_{1}\right)=1$ and $h_{\left(x_{1}, x_{2}\right)}\left(x_{2}\right)=0$. Furthermore, since $X$ is also first countable, we can take $h_{\left(x_{1}, x_{2}\right)}$ such that $h_{\left(x_{1}, x_{2}\right)}^{-1}(\{1\})=\left\{x_{1}\right\}$ and $h_{\left(x_{1}, x_{2}\right)}^{-1}(\{0\})=\left\{x_{2}\right\}$. In particular,

$$
\left|h_{\left(x_{1}, x_{2}\right)}(z)-h_{\left(x_{1}, x_{2}\right)}(w)\right|<\rho\left(h_{\left(x_{1}, x_{2}\right)}\right)
$$

for all $(z, w) \in \tilde{X} \backslash\left\{\left(x_{1}, x_{2}\right),\left(x_{2}, x_{1}\right)\right\}$.

Claim 4. For any $\left(x_{1}, x_{2}\right) \in \tilde{X}$, the set

$$
\mathcal{B}_{\left(x_{1}, x_{2}\right)}=\bigcap_{f \in C(X)} \mathcal{B}_{\left(x_{1}, x_{2}\right), f}
$$

is nonempty, where

$$
\mathcal{B}_{\left(x_{1}, x_{2}\right), f}=\left\{\left(\left(y_{1}, y_{2}\right), \lambda\right) \in \widetilde{Y} \times \mathbb{T}: \Delta(f)\left(y_{1}\right)-\Delta(f)\left(y_{2}\right)=\lambda\left(f\left(x_{1}\right)-f\left(x_{2}\right)\right)\right\} \quad(f \in C(X)) .
$$

Let $\left(x_{1}, x_{2}\right) \in \widetilde{X}$. Given $f \in C(X)$, the set $\mathcal{B}_{\left(x_{1}, x_{2}\right), f}$ is a nonempty subset of $\tilde{Y} \times \mathbb{T}$. Indeed, it suffices to choose $y_{1}, y_{2} \in Y$ such that $\phi_{f, f}\left(y_{i}\right)=x_{i}$ for $i=1,2$. Then

$$
\Delta(f)\left(y_{1}\right)-\Delta(f)\left(y_{2}\right)=\Delta_{f, f}(f)\left(y_{1}\right)-\Delta_{f, f}(f)\left(y_{2}\right)=\lambda_{f, f}\left(f\left(x_{1}\right)-f\left(x_{2}\right)\right),
$$

that is, $\left(\left(y_{1}, y_{2}\right), \lambda_{f, f}\right) \in \mathcal{B}_{\left(x_{1}, x_{2}\right), f}$. An easy verification shows that $\mathcal{B}_{\left(x_{1}, x_{2}\right), f}$ is also closed in $\tilde{Y} \times \mathbb{T}$.

We next prove that the family $\left\{\mathcal{B}_{\left(x_{1}, x_{2}\right), f}: f \in C(X)\right\}$ has the finite intersection property. Let $n \in \mathbb{N}$ and $f_{1}, \ldots, f_{n} \in C(X)$. Take the function $g=h_{\left(x_{1}, x_{2}\right)}$. For each $i \in\{1, \ldots, n\}$, there exists a diameter-preserving linear bijection $\Delta_{g, f_{i}}$ from $C(X)$ to $C(Y)$ such that $\Delta_{g, f_{i}}(g)=\Delta(g)$ and $\Delta_{g, f_{i}}\left(f_{i}\right)=\Delta\left(f_{i}\right)$. Furthermore, we have a homeomorphism $\phi_{g, f_{i}}$ from $Y$ onto $X$, a linear functional $\mu_{g, f_{i}}$ on $C(X)$ and a number $\lambda_{g, f_{i}} \in \mathbb{T}$ with $\lambda_{g, f_{i}} \neq-\mu_{g, f_{i}}\left(1_{X}\right)$ such that

$$
\Delta_{g, f_{i}}(h)(y)=\lambda_{g, f_{i}} h\left(\phi_{g, f_{i}}(y)\right)+\mu_{g, f_{i}}(h) \quad(h \in C(X), y \in Y) .
$$

Let $\left(\left(y_{1}, y_{2}\right), \lambda\right) \in \mathcal{B}_{\left(x_{1}, x_{2}\right), g}$ be arbitrary. For each $i \in\{1, \ldots, n\}$, we obtain

$$
\begin{aligned}
\lambda\left(g\left(x_{1}\right)-g\left(x_{2}\right)\right) & =\Delta(g)\left(y_{1}\right)-\Delta(g)\left(y_{2}\right) \\
& =\Delta_{g, f_{i}}(g)\left(y_{1}\right)-\Delta_{g, f_{i}}(g)\left(y_{2}\right) \\
& =\lambda_{g, f_{i}}\left(g\left(\phi_{g, f_{i}}\left(y_{1}\right)\right)-g\left(\phi_{g, f_{i}}\left(y_{2}\right)\right)\right)
\end{aligned}
$$

and therefore

This implies that either

$$
\left|g\left(\phi_{g, f_{i}}\left(y_{1}\right)\right)-g\left(\phi_{g, f_{i}}\left(y_{2}\right)\right)\right|=1
$$

$$
\left(\phi_{g, f_{i}}\left(y_{1}\right), \phi_{g, f_{i}}\left(y_{2}\right)\right)=\left(x_{1}, x_{2}\right)
$$


or

$$
\left(\phi_{g, f_{i}}\left(y_{1}\right), \phi_{g, f_{i}}\left(y_{2}\right)\right)=\left(x_{2}, x_{1}\right) .
$$

Hence $\lambda_{g, f_{i}}=\lambda$ in the first case, or $\lambda_{g, f_{i}}=-\lambda$ in the second one. We deduce that $\mathcal{B}_{\left(x_{1}, x_{2}\right), g}$ is contained in the set

$$
\left\{\left(\left(\phi_{g, f_{i}}^{-1}\left(x_{1}\right), \phi_{g, f_{i}}^{-1}\left(x_{2}\right)\right), \lambda_{g, f_{i}}\right),\left(\left(\phi_{g, f_{i}}^{-1}\left(x_{2}\right), \phi_{g, f_{i}}^{-1}\left(x_{1}\right)\right),-\lambda_{g, f_{i}}\right)\right\} .
$$

Now, for any $i \in\{1, \ldots, n\}$, we have

$$
\begin{aligned}
\Delta\left(f_{i}\right)\left(y_{1}\right)-\Delta\left(f_{i}\right)\left(y_{2}\right) & =\Delta_{g, f_{i}}\left(f_{i}\right)\left(y_{1}\right)-\Delta_{g, f_{i}}\left(f_{i}\right)\left(y_{2}\right) \\
& =\lambda_{g, f_{i}}\left(f_{i}\left(\phi_{g, f_{i}}\left(y_{1}\right)\right)-f_{i}\left(\phi_{g, f_{i}}\left(y_{2}\right)\right)\right) \\
& =\lambda\left(f_{i}\left(x_{1}\right)-f_{i}\left(x_{2}\right)\right),
\end{aligned}
$$

whence $\left(\left(y_{1}, y_{2}\right), \lambda\right) \in \mathcal{B}_{\left(x_{1}, x_{2}\right), f_{i}}$ and thus

$$
\emptyset \neq \mathcal{B}_{\left(x_{1}, x_{2}\right), g} \subseteq \bigcap_{i=1}^{n} \mathcal{B}_{\left(x_{1}, x_{2}\right), f_{i}} .
$$

This proves that $\left\{\mathcal{B}_{\left(x_{1}, x_{2}\right), f}: f \in C(X)\right\}$ has the finite intersection property, and since $\mathcal{B}_{\left(x_{1}, x_{2}\right), g}$ is a compact subset of $\widetilde{Y} \times \mathbb{T}$, then $\mathcal{B}_{\left(x_{1}, x_{2}\right)}$ will be nonempty.

The proof of Claim 5 is similar to Step 4 of [17].

Claim 5. For every $\left(x_{1}, x_{2}\right) \in \tilde{X}$, there exist $\left(y_{1}, y_{2}\right) \in \tilde{Y}$ and $\lambda \in \mathbb{T}$ such that

$$
\mathcal{B}_{\left(x_{1}, x_{2}\right)}=\left\{\left(\left(y_{1}, y_{2}\right), \lambda\right),\left(\left(y_{2}, y_{1}\right),-\lambda\right)\right\} .
$$

It is immediate from Claim 5 that for every $\left(x_{1}, x_{2}\right) \in \widetilde{X}$, the set

$$
\mathcal{A}_{\left(x_{1}, x_{2}\right)}=\left\{\left(y_{1}, y_{2}\right) \in \widetilde{Y} \mid \exists \lambda \in \mathbb{T}^{+}:\left(\left(y_{1}, y_{2}\right), \lambda\right) \in \mathcal{B}_{\left(x_{1}, x_{2}\right)}\right\}
$$

is a singleton. Let $\Gamma: \widetilde{X} \rightarrow \widetilde{Y}$ be the map given by $\Gamma\left(\left(x_{1}, x_{2}\right)\right)=\left(y_{1}, y_{2}\right)$ where for each $\left(x_{1}, x_{2}\right) \in \widetilde{X}$, the element $\left(y_{1}, y_{2}\right) \in \widetilde{Y}$ is the unique point of $A_{\left(x_{1}, x_{2}\right)}$. We note that if $\mathcal{A}_{\left(x_{1}, x_{2}\right)}=\left\{\left(y_{1}, y_{2}\right)\right\}$, then the definition of $\mathcal{A}_{\left(x_{1}, x_{2}\right)}$ shows that there exists a (unique) scalar $\beta\left(x_{1}, x_{2}\right) \in \mathbb{T}^{+}$, depending on the pair $\left(x_{1}, x_{2}\right)$, such that

$$
\Delta(f)\left(y_{1}\right)-\Delta(f)\left(y_{2}\right)=\beta\left(x_{1}, x_{2}\right)\left(f\left(x_{1}\right)-f\left(x_{2}\right)\right) \quad(f \in C(X)) .
$$

This concludes that

$$
\Delta(f)\left(y_{2}\right)-\Delta(f)\left(y_{1}\right)=\beta\left(x_{1}, x_{2}\right)\left(f\left(x_{2}\right)-f\left(x_{1}\right)\right) \quad(f \in C(X)),
$$

that is $\beta\left(x_{2}, x_{1}\right)=\beta\left(x_{1}, x_{2}\right)$ and $\Gamma\left(\left(x_{2}, x_{1}\right)\right)=\left(y_{2}, y_{1}\right)$.

Claim 6. The map $\Gamma$ is a bijection from $\widetilde{X}$ to $\cup_{\left(x_{1}, x_{2}\right) \in \widetilde{X}} \mathcal{A}_{\left(x_{1}, x_{2}\right)}$.

The surjectivity of $\Gamma$ is immediate, since $\left(y_{1}, y_{2}\right)=\Gamma\left(\left(x_{1}, x_{2}\right)\right)$ if and only if $\left(y_{1}, y_{2}\right) \in \mathcal{A}_{\left(x_{1}, x_{2}\right)}$. To prove its injectivity, let $\left(x_{1}, x_{2}\right),\left(x_{3}, x_{4}\right) \in \widetilde{X}$ be such that

$$
\left(y_{1}, y_{2}\right)=\Gamma\left(\left(x_{1}, x_{2}\right)\right)=\Gamma\left(\left(x_{3}, x_{4}\right)\right) .
$$

Then we have

$$
\beta\left(x_{1}, x_{2}\right)\left(f\left(x_{1}\right)-f\left(x_{2}\right)\right)=\Delta(f)\left(y_{1}\right)-\Delta(f)\left(y_{2}\right)=\beta\left(x_{3}, x_{4}\right)\left(f\left(x_{3}\right)-f\left(x_{4}\right)\right)
$$

for all $f \in C(X)$, where $\beta\left(x_{1}, x_{2}\right), \beta\left(x_{3}, x_{4}\right) \in \mathbb{T}^{+}$. Substituting $f$ by $h_{\left(x_{1}, x_{2}\right)}$, we deduce that $\left\{x_{3}, x_{4}\right\}=\left\{x_{1}, x_{2}\right\}$. Now since both scalars $\beta\left(x_{1}, x_{2}\right)$ and $\beta\left(x_{3}, x_{4}\right)$ are in $\mathbb{T}^{+}$, we get $\left(x_{3}, x_{4}\right)=$ $\left(x_{1}, x_{2}\right)$, as desired.

Claim 7. For any $\left\{x_{1}, x_{2}\right\},\left\{x_{3}, x_{4}\right\} \in X_{2}$, we have

$$
\left|\left\{x_{1}, x_{2}\right\} \cap\left\{x_{3}, x_{4}\right\}\right|=\left|\Lambda_{Y}\left(\Gamma\left(\left(x_{1}, x_{2}\right)\right)\right) \cap \Lambda_{Y}\left(\Gamma\left(\left(x_{3}, x_{4}\right)\right)\right)\right| .
$$


Let $\left\{x_{1}, x_{2}\right\},\left\{x_{3}, x_{4}\right\} \in X_{2}$. If $\left\{x_{1}, x_{2}\right\}=\left\{x_{3}, x_{4}\right\}$, then either $\Gamma\left(\left(x_{1}, x_{2}\right)\right)=\Gamma\left(\left(x_{3}, x_{4}\right)\right)$ or $\Gamma\left(\left(x_{1}, x_{2}\right)\right)=\Gamma\left(\left(x_{4}, x_{3}\right)\right)$ and thus the equality holds. Assume that $\left\{x_{1}, x_{2}\right\} \neq\left\{x_{3}, x_{4}\right\}$. Then $\left(x_{1}, x_{2}\right) \neq\left(x_{3}, x_{4}\right)$ and $\left(x_{1}, x_{2}\right) \neq\left(x_{4}, x_{3}\right)$. Hence $\Gamma\left(\left(x_{1}, x_{2}\right)\right)=\left(y_{1}, y_{2}\right)$ and $\Gamma\left(\left(x_{3}, x_{4}\right)\right)=\left(y_{3}, y_{4}\right)$ for some $\left\{y_{1}, y_{2}\right\},\left\{y_{3}, y_{4}\right\} \in Y_{2}$ with $\left\{y_{1}, y_{2}\right\} \neq\left\{y_{3}, y_{4}\right\}$ by the injectivity of $\Gamma$ and the fact that $\Lambda_{Y}\left(\Gamma\left(\left(x_{1}, x_{2}\right)\right)\right)=\Lambda_{Y}\left(\Gamma\left(\left(x_{2}, x_{1}\right)\right)\right)$. We have two equations:

$$
\begin{aligned}
& \Delta(f)\left(y_{1}\right)-\Delta(f)\left(y_{2}\right)=\beta\left(x_{1}, x_{2}\right)\left(f\left(x_{1}\right)-f\left(x_{2}\right)\right), \\
& \Delta(f)\left(y_{3}\right)-\Delta(f)\left(y_{4}\right)=\beta\left(x_{3}, x_{4}\right)\left(f\left(x_{3}\right)-f\left(x_{4}\right)\right),
\end{aligned}
$$

for all $f \in C(X)$, where $\beta\left(x_{1}, x_{2}\right), \beta\left(x_{3}, x_{4}\right) \in \mathbb{T}^{+}$. Put $g=h_{\left(x_{1}, x_{2}\right)}$ and $h=h_{\left(x_{3}, x_{4}\right)}$. Then using the first equality for $g$ and the second one for $h$, we obtain

$$
\begin{aligned}
& \Delta(g)\left(y_{1}\right)-\Delta(g)\left(y_{2}\right)=\beta\left(x_{1}, x_{2}\right)\left(g\left(x_{1}\right)-g\left(x_{2}\right)\right), \\
& \Delta(h)\left(y_{3}\right)-\Delta(h)\left(y_{4}\right)=\beta\left(x_{3}, x_{4}\right)\left(h\left(x_{3}\right)-h\left(x_{4}\right)\right) .
\end{aligned}
$$

By Claim 2, there exist a homeomorphism $\phi_{g, h}$ from $Y$ onto $X$, a linear functional $\mu_{g, h}$ on $C(X)$ and a number $\lambda_{g, h} \in \mathbb{T}$ with $\lambda_{g, h} \neq-\mu_{g, h}\left(1_{X}\right)$ such that

$$
\Delta(g)(y)=\lambda_{g, h} g\left(\phi_{g, h}(y)\right)+\mu_{g, h}(g)
$$

and

$$
\Delta(h)(y)=\lambda_{g, h} h\left(\phi_{g, h}(y)\right)+\mu_{g, h}(h)
$$

for all $y \in Y$, and therefore

$$
\begin{aligned}
& \Delta(g)\left(y_{1}\right)-\Delta(g)\left(y_{2}\right)=\lambda_{g, h}\left(g\left(\phi_{g, h}\left(y_{1}\right)\right)-g\left(\phi_{g, h}\left(y_{2}\right)\right)\right), \\
& \Delta(h)\left(y_{3}\right)-\Delta(h)\left(y_{4}\right)=\lambda_{g, h}\left(h\left(\phi_{g, h}\left(y_{3}\right)\right)-h\left(\phi_{g, h}\left(y_{4}\right)\right)\right) .
\end{aligned}
$$

It follows that

$$
\begin{gathered}
\lambda_{g, h}\left(g\left(\phi_{g, h}\left(y_{1}\right)\right)-g\left(\phi_{g, h}\left(y_{2}\right)\right)\right)=\beta\left(x_{1}, x_{2}\right)\left(g\left(x_{1}\right)-g\left(x_{2}\right)\right), \\
\lambda_{g, h}\left(h\left(\phi_{g, h}\left(y_{3}\right)\right)-h\left(\phi_{g, h}\left(y_{4}\right)\right)\right)=\beta\left(x_{3}, x_{4}\right)\left(h\left(x_{3}\right)-h\left(x_{4}\right)\right) .
\end{gathered}
$$

These equalities imply that

$$
\left.\left(\phi_{g, h}\left(y_{1}\right), \phi_{g, h}\left(y_{2}\right)\right)\right) \in\left\{\left(x_{1}, x_{2}\right),\left(x_{2}, x_{1}\right)\right\}
$$

and

$$
\left(\phi_{g, h}\left(y_{3}\right), \phi_{g, h}\left(y_{4}\right)\right) \in\left\{\left(x_{3}, x_{4}\right),\left(x_{4}, x_{3}\right)\right\} .
$$

Then we have four possibilities:

(1) $x_{1}=\phi_{g, h}\left(y_{1}\right), x_{2}=\phi_{g, h}\left(y_{2}\right), x_{3}=\phi_{g, h}\left(y_{3}\right), x_{4}=\phi_{g, h}\left(y_{4}\right)$.

(2) $x_{1}=\phi_{g, h}\left(y_{1}\right), x_{2}=\phi_{g, h}\left(y_{2}\right), x_{3}=\phi_{g, h}\left(y_{4}\right), x_{4}=\phi_{g, h}\left(y_{3}\right)$.

(3) $x_{1}=\phi_{g, h}\left(y_{2}\right), x_{2}=\phi_{g, h}\left(y_{1}\right), x_{3}=\phi_{g, h}\left(y_{3}\right), x_{4}=\phi_{g, h}\left(y_{4}\right)$.

(4) $x_{1}=\phi_{g, h}\left(y_{2}\right), x_{2}=\phi_{g, h}\left(y_{1}\right), x_{3}=\phi_{g, h}\left(y_{4}\right), x_{4}=\phi_{g, h}\left(y_{3}\right)$.

If $\left|\left\{x_{1}, x_{2}\right\} \cap\left\{x_{3}, x_{4}\right\}\right|=1$, we infer from injectivity of $\phi_{g, h}$ that

$$
\left|\Lambda_{Y}\left(\Gamma\left(\left(x_{1}, x_{2}\right)\right)\right) \cap \Lambda_{Y}\left(\Gamma\left(\left(x_{3}, x_{4}\right)\right)\right)\right|=\left|\left\{y_{1}, y_{2}\right\} \cap\left\{y_{3}, y_{4}\right\}\right|=1,
$$

while if $\left|\left\{x_{1}, x_{2}\right\} \cap\left\{x_{3}, x_{4}\right\}\right|=0$, then

$$
\left|\Lambda_{Y}\left(\Gamma\left(\left(x_{1}, x_{2}\right)\right)\right) \cap \Gamma\left(\left(x_{3}, x_{4}\right)\right)\right|=\left|\left\{y_{1}, y_{2}\right\} \cap\left\{y_{3}, y_{4}\right\}\right|=0 .
$$

The proof of Claim 8 is the same as that of Step 10 of [17].

Claim 8. Assume $|X| \geq 3$. For each $x \in X$ and any $\left\{x_{1}, x_{2}\right\} \in X_{2}$ with $x_{1} \neq x \neq x_{2}$, there exists a unique point, depending only on $x$ and denoted by $\varphi(x)$, in the intersection $\Gamma\left(\left\{x, x_{1}\right\}\right) \cap \Gamma\left(\left\{x, x_{2}\right\}\right)$. Then the map $\varphi: X \rightarrow Y$ is injective and $\left\{\varphi\left(x_{1}\right), \varphi\left(x_{2}\right)\right\}=\Lambda_{Y}\left(\Gamma\left(\left(x_{1}, x_{2}\right)\right)\right)$ for all $\left\{x_{1}, x_{2}\right\} \in X_{2}$. 
Let $Y_{0}=\varphi(X)$. Since the map $\varphi: X \rightarrow Y$ is injective, its inverse $\phi_{0}: Y_{0} \rightarrow X$ is a bijection which satisfies

$$
\left\{y_{1}, y_{2}\right\}=\Lambda_{Y}\left(\Gamma\left(\left(\phi_{0}\left(y_{1}\right), \phi_{0}\left(y_{2}\right)\right)\right) \quad\left(\left\{y_{1}, y_{2}\right\} \in\left(Y_{0}\right)_{2}\right) .\right.
$$

Now the same argument as in Step 12 of [17] yields the next claim.

Claim 9. There exists a number $\lambda \in \mathbb{T}$ such that

$$
\Delta(f)\left(y_{1}\right)-\Delta(f)\left(y_{2}\right)=\lambda\left(f\left(\phi_{0}\left(y_{1}\right)\right)-f\left(\phi_{0}\left(y_{2}\right)\right)\right) \quad\left(f \in C(X), y_{1}, y_{2} \in Y_{0}\right) .
$$

Using the above claim we can define a functional $\mu: C(X) \rightarrow \mathbb{C}$ by

$$
\mu(f)=\Delta(f)\left(y_{0}\right)-\lambda f\left(\phi_{0}\left(y_{0}\right)\right) \quad(f \in C(X)),
$$

where $y_{0}$ is an arbitrary point of $Y_{0}$. Then it is obvious that $\mu$ is well-defined and homogeneous and, moreover,

$$
\Delta(f)(y)=\lambda f\left(\phi_{0}(y)\right)+\mu(f) \quad\left(f \in C(X), y \in Y_{0}\right) .
$$

Note that $\Delta\left(1_{X}\right)$ is a nonzero constant function by Claim [3. Hence it follows from (11) that $\mu\left(1_{X}\right) \neq-\lambda$.

The proof of Step 15 of [17] can be applied to get the next claim.

Claim 10. $\phi_{0}: Y_{0} \rightarrow X$ is a homeomorphism.

In the next claims we shall show that the homeomorphism $\phi_{0}: Y_{0} \rightarrow X$ can be extended to a homeomorphism $\phi: Y \rightarrow X$ satisfying $\Delta(f)(y)=\lambda f(\phi(y))+\mu(f)$ for all $f \in C(X)$ and $y \in Y$. To do this we first prove the next claim.

Claim 11. The map $S: C(X) \rightarrow C(Y)$ defined by

$$
S(f)(y)=\lambda^{-1}(\Delta(f)(y)-\mu(f)) \quad(f \in C(X), y \in Y)
$$

is a unital algebra homomorphism.

Fix a point $y \in Y$ and define the functional $S_{y}: C(X) \rightarrow \mathbb{C}$ by

$$
S_{y}(f)=\lambda^{-1}(\Delta(f)(y)-\mu(f)) \quad(f \in C(X)) .
$$

Since $\Delta\left(1_{X}\right)$ is a constant function it follows from the equality (1) that $S_{y}\left(1_{X}\right)=1$. We next prove that $S_{y}$ is linear and multiplicative. Since $S_{y}\left(0_{X}\right)=0$, by the Kowalski-Słodkowski theorem [19] it suffices to show that $S_{y}(f)-S_{y}(g) \in(f-g)(X)$ for every $f, g \in C(X)$. Let $f, g \in C(X)$. Since $\phi_{0}: Y_{0} \rightarrow X$ is a bijective map, there exists $y_{0} \in Y_{0}$ such that $\phi_{0}\left(y_{0}\right)=\phi_{f, g}(y)$. Construct the sequence $\left\{y_{i}\right\}_{i=0}^{\infty}$ in $Y_{0}$ such that

$$
\phi_{0}\left(y_{i+1}\right)=\phi_{f, g}\left(y_{i}\right) \quad(i \in \mathbb{N} \cup\{0\}) .
$$

Since $Y_{0}$ is a first countable compact Hausdorff space, passing through a subsequence we may assume that $\left\{y_{i}\right\}_{i} \rightarrow z_{0}$ for some $z_{0} \in Y_{0}$. Hence, tending $i \rightarrow \infty$ in the above equality, we get $\phi_{0}\left(z_{0}\right)=\phi_{f, g}\left(z_{0}\right)$. Since $z_{0}, y_{i} \in Y_{0}$, Claim 9 provides the equations:

$$
\Delta(f)\left(z_{0}\right)-\Delta(f)\left(y_{i}\right)=\lambda\left(f\left(\phi_{0}\left(z_{0}\right)\right)-f\left(\phi_{0}\left(y_{i}\right)\right)\right)
$$

and

$$
\Delta(g)\left(z_{0}\right)-\Delta(g)\left(y_{i}\right)=\lambda\left(g\left(\phi_{0}\left(z_{0}\right)\right)-g\left(\phi_{0}\left(y_{i}\right)\right)\right) .
$$

On the other hand, since $\phi_{f, g}\left(z_{0}\right)=\phi_{0}\left(z_{0}\right)$ and $\phi_{f, g}\left(y_{i}\right)=\phi_{0}\left(y_{i+1}\right)$, we have

$$
\begin{aligned}
\Delta(f)\left(z_{0}\right)-\Delta(f)\left(y_{i}\right) & =\lambda_{f, g}\left(f\left(\phi_{f, g}\left(z_{0}\right)\right)-f\left(\phi_{f, g}\left(y_{i}\right)\right)\right) \\
& =\lambda_{f, g}\left(f\left(\phi_{0}\left(z_{0}\right)\right)-f\left(\phi_{0}\left(y_{i+1}\right)\right)\right)
\end{aligned}
$$

and

$$
\begin{aligned}
\Delta(g)\left(z_{0}\right)-\Delta(g)\left(y_{i}\right) & =\lambda_{f, g}\left(g\left(\phi_{f, g}\left(z_{0}\right)\right)-g\left(\phi_{f, g}\left(y_{i}\right)\right)\right) \\
& =\lambda_{f, g}\left(g\left(\phi_{0}\left(z_{0}\right)\right)-g\left(\phi_{0}\left(y_{i+1}\right)\right)\right) .
\end{aligned}
$$


Hence, using the cited equations above, for each $i \in \mathbb{N} \cup\{0\}$ we have

$$
f\left(\phi_{0}\left(z_{0}\right)\right)-f\left(\phi_{0}\left(y_{i}\right)\right)=\lambda^{-1} \lambda_{f, g}\left(f\left(\phi_{0}\left(z_{0}\right)\right)-f\left(\phi_{0}\left(y_{i+1}\right)\right)\right)
$$

and

$$
g\left(\phi_{0}\left(z_{0}\right)\right)-g\left(\phi_{0}\left(y_{i}\right)\right)=\lambda^{-1} \lambda_{f, g}\left(g\left(\phi_{0}\left(z_{0}\right)\right)-g\left(\phi_{0}\left(y_{i+1}\right)\right)\right) .
$$

Now, it follows by induction that for each $i \in \mathbb{N} \cup\{0\}$ and $n \in \mathbb{N}$, we have

$$
f\left(\phi_{0}\left(z_{0}\right)\right)-f\left(\phi_{0}\left(y_{i}\right)\right)=\left(\lambda^{-1} \lambda_{f, g}\right)^{n}\left(f\left(\phi_{0}\left(z_{0}\right)\right)-f\left(\phi_{0}\left(y_{i+n}\right)\right)\right),
$$

and

$$
g\left(\phi_{0}\left(z_{0}\right)\right)-g\left(\phi_{0}\left(y_{i}\right)\right)=\left(\lambda^{-1} \lambda_{f, g}\right)^{n}\left(g\left(\phi_{0}\left(z_{0}\right)\right)-g\left(\phi_{0}\left(y_{i+n}\right)\right)\right),
$$

Thus tending $n \rightarrow \infty$ above, we get

$$
f\left(\phi_{0}\left(z_{0}\right)\right)=f\left(\phi_{0}\left(y_{i}\right)\right) \quad(i \in \mathbb{N} \cup\{0\}) .
$$

and

$$
g\left(\phi_{0}\left(z_{0}\right)\right)=g\left(\phi_{0}\left(y_{i}\right)\right) \quad(i \in \mathbb{N} \cup\{0\}) .
$$

Therefore, for each $i \in \mathbb{N} \cup\{0\}$, we infer from the equations that

$$
\Delta(f)\left(z_{0}\right)-\Delta(f)\left(y_{i}\right)=\lambda\left(f\left(\phi_{0}\left(z_{0}\right)\right)-f\left(\phi_{0}\left(y_{i}\right)\right)\right)=0,
$$

and

$$
\Delta(g)\left(z_{0}\right)-\Delta(g)\left(y_{i}\right)=\lambda\left(g\left(\phi_{0}\left(z_{0}\right)\right)-g\left(\phi_{0}\left(y_{i}\right)\right)\right)=0,
$$

that is,

$$
\Delta(f)\left(z_{0}\right)=\Delta(f)\left(y_{i}\right), \quad \Delta(g)\left(z_{0}\right)=\Delta(g)\left(y_{i}\right) \quad(i \in \mathbb{N} \cup\{0\}),
$$

and taking limits with $i \rightarrow \infty$ above, we deduce that

$$
\Delta(f)\left(z_{0}\right)=\Delta(f)\left(y_{0}\right), \quad \Delta(g)\left(z_{0}\right)=\Delta(g)\left(y_{0}\right) .
$$

On the other hand, notice that $f\left(\phi_{f, g}(y)\right)=f\left(\phi_{0}\left(y_{0}\right)\right)=f\left(\phi_{0}\left(z_{0}\right)\right)$, and consequently

$$
\begin{aligned}
\Delta(f)(y) & =\lambda_{f, g} f\left(\phi_{f, g}(y)\right)+\mu_{f, g}(f) \\
& =\lambda_{f, g} f\left(\phi_{0}\left(z_{0}\right)\right)+\mu_{f, g}(f) \\
& =\lambda_{f, g} f\left(\phi_{f, g}\left(z_{0}\right)\right)+\mu_{f, g}(f) \\
& =\Delta(f)\left(z_{0}\right) .
\end{aligned}
$$

Therefore we have

$$
\Delta(f)(y)=\Delta(f)\left(z_{0}\right)=\Delta(f)\left(y_{0}\right)
$$

and, similarly, we can obtain

$$
\Delta(g)(y)=\Delta(g)\left(z_{0}\right)=\Delta(g)\left(y_{0}\right) .
$$

Now, using the equality (11) and the definition of $S_{y}$, we can write

$$
\begin{aligned}
& \Delta(f)\left(y_{0}\right)=\lambda f\left(\phi_{0}\left(y_{0}\right)\right)+\mu(f)=\lambda f\left(\phi_{0}\left(y_{0}\right)\right)+\Delta(f)(y)-\lambda S_{y}(f), \\
& \Delta(g)\left(y_{0}\right)=\lambda g\left(\phi_{0}\left(y_{0}\right)\right)+\mu(g)=\lambda g\left(\phi_{0}\left(y_{0}\right)\right)+\Delta(g)(y)-\lambda S_{y}(g),
\end{aligned}
$$

which imply

$$
\begin{gathered}
S_{y}(f)=f\left(\phi_{0}\left(y_{0}\right)\right)+\lambda^{-1}\left(\Delta(f)(y)-\Delta(f)\left(y_{0}\right)\right)=f\left(\phi_{0}\left(y_{0}\right)\right), \\
S_{y}(g)=g\left(\phi_{0}\left(y_{0}\right)\right)+\lambda^{-1}\left(\Delta(g)(y)-\Delta(g)\left(y_{0}\right)\right)=g\left(\phi_{0}\left(y_{0}\right)\right) .
\end{gathered}
$$

Finally, we deduce the required condition:

$$
S_{y}(f)-S_{y}(g)=f\left(\phi_{0}\left(y_{0}\right)\right)-g\left(\phi_{0}\left(y_{0}\right)\right) \in(f-g)(X) .
$$

Hence $S_{y}$ is a unital multiplicative linear functional on $C(X)$. Since $y$ was arbitrary, we conclude that $S: C(X) \rightarrow C(Y)$ is a unital algebra homomorphism. 
Claim 12. There exists a homeomorphism $\phi: Y \rightarrow X$ such that

$$
\Delta(f)=\lambda f \circ \phi+\mu(f) 1_{Y} \quad(f \in C(X)) .
$$

Let $S: C(X) \rightarrow C(Y)$ be the unital algebra homomorphism given in Claim [1] By Gelfand theory, $S$ induces a continuous map $\phi: Y \rightarrow X$ such that $S(f)=f \circ \phi$ for all $f \in C(X)$, and thus $\Delta(f)=\lambda f \circ \phi+\mu(f) 1_{Y}$ for all $f \in C(X)$. Now, a similar proof to that of Step 17 in [17] shows that $\phi$ is a homeomorphism from $Y$ onto $X$.

We note that $\phi(y)=\phi_{0}(y)$ for all $y \in Y_{0}$, since by Claim 12 and the equation (11) we have $f(\phi(y))=f\left(\phi_{0}(y)\right)$ for all $f \in C(X)$ and $y \in Y_{0}$.

Claim 13. For each $f \in C(X)$, we have $T\left(\pi_{X}(f)\right)=\pi_{Y}(\lambda f \circ \phi)$. In particular, $T$ is linear and surjective.

Let $f \in C(X)$. By Claim 12 and the definition of $\Delta$, we have

$$
\lambda f \circ \phi+\mu(f) 1_{Y}=\Delta(f)=\Psi_{Y}^{-1}\left(T\left(\pi_{X}(f)\right), f\left(u_{0}\right)\right) .
$$

Hence $\Psi_{Y}\left(\lambda f \circ \phi+\mu(f) 1_{Y}\right)=\left(T\left(\pi_{X}(f)\right), f\left(u_{0}\right)\right)$ which implies

$$
\pi_{Y}(\lambda f \circ \phi)=\pi_{Y}\left(\lambda f \circ \phi+\mu(f) 1_{Y}\right)=T\left(\pi_{X}(f)\right) .
$$

This completes the proof of Theorem 2 .

Acknowledgements. Research partially supported by Junta de Andalucía grant FQM194.

\section{REFERENCES}

[1] A. Aizpuru and F. Rambla, There's something about the diameter, J. Math. Anal. Appl. 330 (2007), 949-962.

[2] A. Aizpuru and F. Rambla, Diameter preserving bijections and $C_{0}(L)$ spaces, Bull. Belg. Math. Soc. Simons 17 (2010), 377-383.

[3] A. Aizpuru and M. Tamayo, Linear bijections which preserve the diameter of vector-valued maps, Linear Algebra Appl. 424 (2007), 371-377.

[4] B. A. Barnes and A. K. Roy, Diameter preserving maps on various classes of function spaces, Studia Math. 153 (2002), 127-145.

[5] F. Cabello Sánchez, Diameter preserving linear maps and isometries, Arch. Math. (Basel) 73 (1999), 373-379.

[6] F. Cabello Sánchez and L. Molnár, Reflexivity of the isometry group of some classical spaces, Rev. Mat. Iberoamericana, 18, no. 2 (2002), 409-430.

[7] J. J. Font and M. Hosseini, Diameter preserving maps on function spaces, Positivity 21 (2017), no. 3, 875-883.

[8] J. J. Font and M. Hosseini (2019): Nonlinear diameter preserving maps on function spaces, Quaestiones Mathematicae, DOI: 10.2989/16073606.2018.1536896

[9] J. J. Font and M. Sanchís, Extreme points and the diameter norm, Rocky Mountain J. Math. 34 (2004), 13251331.

[10] F. González and V. V. Uspenskij, On homomorphisms of groups of integer-valued functions, Extracta Math. 14 (1999), 19-29.

[11] M. Győry, 2-local isometries of $C_{0}(X)$, Acta Sci. Math. (Szeged) 67 (2001), 735-746.

[12] M. Győry and L. Molnár, Diameter preserving linear bijections of $C(X)$, Arch. Math. (Basel) 71 (1998), 301-310.

[13] O. Hatori, T. Miura, H. Oka and H. Takagi, 2-local isometries and 2-local automorphisms on uniform algebras, Int. Math. Forum 50 (2007), 2491-2502.

[14] O. Hatori and S. Oi, 2-local isometries on functions spaces, Recent trends in operator theory and applications, 89-106, Contemp. Math., 737, Amer. Math. Soc., Providence, RI, 2019.

[15] M. Hosseini, Generalized 2-local isometries of spaces of continuously differentiable functions, Quaest. Math. 40 (2017), 1003-1014.

[16] A. Jamshidi and F. Sady, Nonlinear diameter preserving maps between certain function spaces, Mediterr. J. Math. 13 (2016), 4237-4251.

[17] A. Jiménez-Vargas and F. Sady, Algebraic reflexivity of diameter-preserving linear bijections between $C(X)$ spaces, arXiv:2004.05864.

[18] A. Jimenez-Vargas and M. Villegas-Vallecillos, 2-local isometries on spaces of Lipschitz functions, Canad. Math. Bull. 54 (2011), 680-692.

[19] S. Kowalski and Z. Słodkowski, A characterization of multiplicative linear functionals in Banach algebras, Studia Math. 67 (1980), pp. 215-223.

[20] L. Li, A. M. Peralta, L. Wang and Y.-S. Wang, Weak-2-local isometries on uniform algebras and Lipschitz algebras, Publ. Mat. 63 (2019), 241-264. 
[21] L. Molnár, 2-local isometries of some operator algebras, Proc. Edinburgh Math. 45 (2002), 349-352.

[22] L. Molnár and B. Zalar, Reflexivity of the group of surjective isometries of some Banach spaces, Proc. Edin. Math. Soc. 42 (1999), 17-36.

[23] T. S. S. R. K. Rao and A. K. Roy, Diameter preserving linear bijections of function spaces, J. Aust. Math. Soc. 70 (2001), 323-335.

Departamento de Matemáticas, Universidad de Almería, 04120, Almeria, Spain

E-mail address: ajimenez@ual.es

Department of Pure Mathematics, Faculty of Mathematical Sciences, Tarbiat Modares University, TEHRAN 14115-134, IRAN

E-mail address: sady@modares.ac.ir 